\title{
TAMANHO DE AMOSTRA NA PARCELA PARA CARACTERIZAÇÃO DA ALTURA DE PLANTAS DE ALGODOEIRO HERBÁCEO Gossypium hirsutum
}

\author{
SAMPLE SIZE IN THE PLOT FOR CHARACTERIZATION OF THE \\ Gossypium hirsutum HEIGHT
}

\author{
Joelson André de Freitas ${ }^{1}$ Enilson de Barros Silva ${ }^{2}$ Joel Fallieri $^{3}$ \\ Marcelo Abreu Lanza ${ }^{4}$ Renato Soares de Faria ${ }^{5}$ Petrônio José da Silva ${ }^{5}$
}

RESUMO

\begin{abstract}
Dados da altura individual de dez plantas, obtidos de dez experimentos instalados em blocos casualizados com quatro repetições e quatro cultivares de algodoeiro herbáceo, foram estudados, para determinação do número mínimo de plantas/parcela, necessárias para caracterização da altura média de plantas de algodoeiro herbáceo. Foram estudados cinco tamanhos de amostra na parcela, cada uma, contendo $2,4,6,8$ e 10 plantas. Os coeficientes de variação experimental e amostral e as significâncias observadas nas análises de variâncias, indicaram que parcelas contendo seis ou mais plantas, permitiram boa caracterização do porte médio das variedades de algodoeiro testadas. Os resultados sugerem, portanto que, no processo de caracterização da altura média do algodoeiro herbáceo, a avaliação pode ser mais rápida, além de haver uma redução na mão-de-obra de coleta e manuseio dos dados, em decorrência de uma diminuição do número de plantas amostradas na parcela.
\end{abstract}

Palavras-chave: amostragem, coeficiente de variação.

SUMMARY

Data of measurements of ten individual plants height, from ten randomized blocks design experiments, with four replications and four cotton cultivars, were evaluated to determine the minimum number of plants per plot for characterization of the average height in cotton. To reach the purpose of this work, five sample sizes in the plot were studied, with 2, 4, 6, 8 and 10 plants each. The coefficients of experimental and amostral variation, as well as the significative analysis of variances, indicated that plots with six or more plants allowed a good characterization of the plant height. The results showed that in the characterization of the height of the gossypium plants, the evaluating become fast and it can have a reduction in the labor during the collection and handling of the data, due to the reduction of the number of plants sampled in the plot.

Key words: sampling, coefficient of variation.

\section{INTRODUÇÃO}

A experimentação agrícola no melhoramento de plantas, assim como em outras áreas correlatas, deve ser bem conduzida, pois a precisão experimental caracteriza a qualidade da inferência nos resultados. Tal precisão pode ser melhorada, quando se consegue amenizar alguns efeitos como temperatura, umidade e solo. Destes, o efeito da heterogeneidade do solo, fator possível de ser manipulado, é o que mais prejudica a precisão de um experimento (GOMES \& GOMES, 1984). Vários mecanismos são adotados para se minimizar o erro experimental, a despeito da heterogeneidade do solo, como a utilização de um delineamento adequado e minucioso critério de escolha do material experimental, condução do experimento e coleta dos dados (FERH, 1987).

A manipulação do número de repetições e do tamanho da parcela também são importantes estratégias para reduzir o erro experimental. A parcela, unidade básica do experimento, deve ser capaz de reduzir ao máximo o efeito da heterogeneidade ambiental e a variabilidade genética do material experimental. Por influenciar diretamente os gastos com recursos disponíveis à condução dos experi-

\footnotetext{
${ }^{1}$ Engenheiro Agrônomo, Doutor, Genética e Melhoramento de Plantas, EPAMIG/CTNM, CP 12, 39440-000, Janaúba, MG. E-mail: freitasja@nortecnet.com.br. Autor para correspondência.

${ }^{2}$ Engenheiro Agrônomo .Doutor, Nutrição de Plantas, EPAMIG/CTNM, Janaúba, MG

${ }^{3}$ Engenheiro Agrônomo, PhD., Melhoramento Vegetal, EMBRAPA-EPAMIG/CTTP, Uberaba, MG.

${ }^{4}$ Engenheiro Agrônomo, MSc., Fitoquímica, EPAMIG/CTTP, Uberaba, MG.

${ }^{5}$ Técnicos em Agropecuária, Nível Médio, EPAMIG/CTNM e CTTP. 
mentos (STEELL \& TORRIE, 1960), sejam eles insumos ou mão-de-obra, estudos sobre tamanho de parcela frequentemente são relatados (IGUE $\boldsymbol{e t} \boldsymbol{a l}$., 1972; CORDEIRO et al., 1982 e CHAVES \& MIRANDA FILHO, 1992). Estudos sobre tamanho de parcela têm contribuído sobremaneira para a redução de recursos financeiros empregados na pesquisa, a exemplo dos resultados obtidos por VENEGAS $\boldsymbol{e}$ t al. (1980), BERTOLUCCI (1990) e VARGAS (1996) para as culturas do milho, café e feijão, respectivamente, dentre outros.

Além do melhor tamanho de parcela, o conhecimento do melhor tamanho de amostra também tem relevada importância, quando se deseja reduzir os custos na pesquisa, permanecendo pois, baixos, os erros cometidos nas inferências. Métodos clássicos de amostragem são descritos em COCHRAN (1977), embora, cada peculiaridade de trabalho, exija estudos apropriados quanto ao tamanho de amostra. SANTANA et al. (1997) compararam os métodos de amostragem fixa e sequencial, concluindo que este último se aplicou melhor quando os objetivos foram redução de tempo e sementes gastas, na avaliação da porcentagem de germinação de sementes de milho.

No caso da experimentação com algodoeiro Gossypium hirsutum, a altura de plantas é uma característica importante, observada na caracterização de cultivares melhoradas, onde se deseja aquelas que aliem menor porte, visando facilitar a colheita mecânica, pois plantas muito altas tendem a acamar. Atualmente, o tamanho da parcela útil nos experimentos com a cultura, utilizado no programa de melhoramento do algodoeiro na EPAMIG, consta de 50 plantas (2 fileiras de $5 \mathrm{~m}$ de comprimento), para coleta dos dados de produtividade, precocidade de produção e resistência a doenças. Para as avaliações do peso médio de capulho (1 capulho/planta), data de florescimento e altura de plantas, são amostrados na parcela útil, 20, 2 e 10 plantas, respectivamente. Quanto ao tamanho de amostra utilizado no programa de melhoramento do algodoeiro da EPAMIG (10 plantas/parcela), para determinar a altura média de plantas, tem se verificado boa eficiência na caracterização de genótipos em experimentos de campo com 4 ou 5 repetições (FALLIERI, 1994). BARREIRO NETO et al. (1982) também determinaram com sucesso, a altura média da cultivar 'Acala Del Cerro', amostrando 10 plantas em um total de 80 , dispostas em duas fileiras de $20 \mathrm{~m}$ de comprimento.

Como a obtenção da altura de plantas é uma operação que contribui para aumentar o dispêndio com mão-de-obra, além do acréscimo da massa de dados a serem trabalhados matematicamente, tem-se por objetivo neste estudo, verificar, se existe possibilidade de redução do tamanho da amostra na parcela, permanecendo pois, precisas, as inferências estatísticas sobre a estimativa da altura média de plantas de algodoeiro.

\section{MATERIAL E MÉTODOS}

Utilizaram-se os dados da altura individual de plantas de algodoeiro herbáceo, obtidos de dez experimentos conduzidos em cinco épocas de plantio e duas safras agrícolas. As cultivares 'Epamig precoce-1', 'Redenção', 'Alva' e 'Liça', foram avaliadas quanto à altura média das plantas, instalando-se para este fim, os experimentos 1: $1^{\underline{a}}$ quinzena de novembro, 2: $2^{\mathrm{a}}$ quinzena de novembro, 3: $1^{\mathrm{a}}$ quinzena de dezembro, 4: $2^{\mathrm{a}}$ quinzena de dezembro e 5: $1^{\text {a }}$ quinzena de janeiro, nas safras agrícolas 1997/98 e 1998/99. Os ensaios foram conduzidos na Fazenda Experimental do Gorutuba EPAMIG/CTNM, município de Nova Porteirinha, MG (altitude $515 \mathrm{~m}$, latitude $15^{0} 47^{\prime} \mathrm{S}$ e longitude $43^{0} 18^{\prime} \mathrm{W}$ ), em solos de texturas franco argiloso siltoso (safra 1997/98) e franco arenosa (safra 1998/99).

Os dez experimentos foram instalados em blocos casualizados com 4 repetições e 50 plantas/parcela útil, em que se obteve as alturas de 10 plantas amostradas dentre as 50. Este número de plantas amostradas na parcela foi estabelecido, tendo em vista que 10 plantas têm sido utilizadas com sucesso nos trabalhos do programa de melhoramento de algodoeiro da EPAMIG (FALLIERI, 1994) e que se deseja verificar, se este número poderia ser ainda reduzido, para 8, 6, 4 ou 2 plantas/parcela. Em todos os experimentos foram feitas adubações de plantio e cobertura, conforme resultados da análise dos solos e recomendações para a cultura (COMISSÃO..., 1989), assim como o controle químico de insetospraga e doenças, quando necessário. As alturas das plantas foram obtidas a 110 dias após a semeadura, medindo-se seu comprimento, do colo até a última gema apical, com fita métrica graduada em centímetros.

Inicialmente, foi aplicado o teste da razão máxima (Hartley) (RAMALHO et al., 2000), para verificar a hipótese da homogeneidade de variância dos erros amostrais entre os experimentos e decidir sobre a conveniência de uma análise conjunta dos experimentos. $\mathrm{O}$ teste foi aplicado para verificar a possibilidade de análise conjunta dos cinco experimentos (cinco épocas de plantio), para todos os tamanhos de amostra $(2,4,6,8$ e 10 plantas/parcela), em cada uma das safras agrícolas compreendidas (1997/98 e 1998/99). Para a aplicação desse teste, primeiro, foram feitas as análises de variâncias individuais de cada experimento. De posse dos valores extremos dos QMerros amostrais, considerando ambos os cinco 
experimentos das épocas de plantio em cada safra agrícola e, para cada tamanho de amostra, foram calculados os valores de Hartley (Hc). Estes foram comparados com os valores tabelados ao nível de $1 \%(\mathrm{Ht})$, obtidos considerando os parâmetros $\mathrm{v}_{1}$ $\left(=\mathrm{n}^{\mathrm{o}}\right.$ de experimentos envolvidos $)$ e $\mathrm{v}_{2}\left(=\mathrm{n}^{\mathrm{o}}\right.$ de graus de liberdade associado ao erro amostral). Também de posse das análises de variâncias foram obtidas as esperanças dos quadrados médios, para cálculo dos coeficientes de variação experimental (C.V.exp. $)$ e amostral (C.V.amostr. $)$ como se segue:

C.V.exp. $=100\left[\left(\hat{\sigma}_{e}^{2}\right)^{0,5}\right] / \hat{m}$;

C. $V_{\text {amostr. }}=100\left[\left(\text { QMerro }_{\text {amostr. }}\right)^{0,5}\right] / \hat{m} ; \quad$ onde,

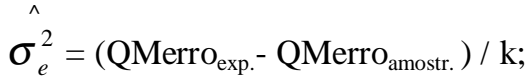

$\hat{m}$ : é a estimativa da média geral de cada experimento com $\mathrm{k}$ amostras; e

$\mathrm{k}$ : número de plantas amostradas na parcela $(\mathrm{k}=2,4$, $6,8$ e 10$)$.

Os dados não sofreram nenhum tipo de transformação e as análises de variâncias foram feitas utilizando-se o procedimento PROC ANOVA do software estatístico Statistical Analysis System (SAS) for windows.

\section{RESULTADOS E DISCUSSÃO}

O teste de Hartley foi significativo ao nível de $1 \%$ em alguns casos (Tabela 1) indicando a

Tabela 1 - Teste de Hartley para verificação da homogeneidade das variância dos erros amostrais, entre cinco experimentos de caracterização da altura de plantas de cultivares de algodoeiro herbáceo, conduzidos em duas safras. EPAMIG/CTNM. Nova Porteirinha, MG, 2000.

\begin{tabular}{|c|c|c|c|c|c|c|}
\hline \multirow[t]{2}{*}{ Safra } & \multirow{2}{*}{$\begin{array}{c}\text { Variâncias } \\
\text { (5 experimentos) }\end{array}$} & \multicolumn{5}{|c|}{$\mathrm{N}^{\mathrm{o}}$ de plantas amostradas na parcela (n) } \\
\hline & & 2 & 4 & 6 & 8 & 10 \\
\hline \multirow[t]{4}{*}{1998} & Var. máx & 175,75 & 130,67 & 104,71 & 99,53 & 105,11 \\
\hline & Var. mín & 39,28 & 34,22 & 34,65 & 32,54 & 33,05 \\
\hline & ${ }^{1 /} \mathrm{Hc}$ & $4,47^{\mathrm{ns}}$ & $3,82^{* *}$ & $3,02^{* *}$ & $3,06^{* *}$ & $3,18^{* *}$ \\
\hline & $\left.{ }^{2 /} \mathrm{Ht}_{(\mathrm{v} 1 \mathrm{e}} \mathrm{v} 2\right) \propto$ & 5,72 & 2,80 & 1,00 & 1,00 & 1,00 \\
\hline \multirow[t]{4}{*}{1999} & Var. máx & 386,09 & 266,57 & 246,86 & 285,53 & 285,53 \\
\hline & Var. mín & 162,56 & 125,71 & 137,75 & 123,99 & 123,99 \\
\hline & ${ }^{1 /} \mathrm{Hc}$ & $2,38^{\mathrm{ns}}$ & $2,12^{\mathrm{ns}}$ & $1,79^{* *}$ & $2,30^{* *}$ & $2,30^{* *}$ \\
\hline & $\left.{ }^{2 /} \mathrm{Ht}_{(\mathrm{v} 1 \mathrm{e}} \mathrm{v} 2\right) \propto$ & 5,72 & 2,80 & 1,00 & 1,00 & 1,00 \\
\hline
\end{tabular}

${ }^{1 /} \mathrm{e}^{2 /}$ : valores de Hartley calculados e tabelados (com $\mathrm{v}_{1}$ experimentos, $\mathrm{v}_{2}$ graus de liberdade dos erros amostrais e $\propto=1 \%$ ), respectivamente; ${ }^{\text {ns/ }}$ : não significativo;

${ }^{* *}$ : rejeitada a hipótese de homogeneidade das variâncias dos erros amostrais entre os experimentos, em nível de $1 \%$. não homogeneidade das variâncias dos erros amostrais entre os experimentos das safras 1997/98 (análises com 4, 6, 8 e 10 plantas/parcela) e 1998/99 (análises com 6, 8 e 10 plantas/parcela). Apenas as análises dos experimentos de 1997/98 (com 2 plantas/parcela) e 1998/99 (com 2 e 4 plantas/parcela), apresentaram homogeneidade das variâncias. Por isto, decidiu-se realizar as inferências com base nas análises individuais de cada experimento.

Analisando os resultados da tabela 2, quando se realizou amostragem de 2 plantas/parcela, apenas três dos dez experimentos (4/98, 2/99 e 5/99) apresentaram significâncias da fonte de variação 'Cultivares'. Tomando-se dados de altura de 4 plantas/parcela para compor as análises, o efeito de 'Cultivares' foi significativo em seis dos dez experimentos (1/98, 2/98, 4/98, 1/99, 2/99 e 5/99). Quando as análises foram feitas com 6 plantas/parcela verificou-se significância dessa fonte de variação em oito dos dez experimentos, enquanto que com 8 plantas/parcela todos os experimentos mostraram significância do efeito de 'Cultivares'. Estes resultados indicam que quando o objetivo maior for reduzir trabalho, em experimentos em blocos casualizados com 4 repetições, pode-se optar por amostrar 4 plantas em parcela de 50 plantas (2 fileiras de $5 \mathrm{~m}$ de comprimento). Todavia, maior segurança nas inferências pode ser obtida, ainda com uma redução de trabalho, amostrando-se 6 ou 8 plantas/parcela. Quando foram usados os dados das 10 plantas/parcela, um dos dez experimentos realizados (3/98) não apresentou significância do efeito de 'Cultivares'; ainda assim, pode-se dizer, que o tamanho de amostra na parcela (10 plantas), usualmente adotado para determinar a altura de algodoeiro no programa de pesquisa da EPAMIG, é adequado, em conformidade com BARREIRO NETO et al. (1982).

Os QMerros experimental e amostral (Tabela 2) foram obtidos especialmente para o cálculo dos coeficientes de variação experimental e amostral, respectivamente. No entanto, de maneira geral para os dez experimentos, mas considerando-se em separado cada tamanho de amostra testado, ficou constatado a significância do erro experimental. Apenas na situação onde foram amostradas 2 plantas/parcela, o erro experimental foi significativo em três dos dez experimentos (3/98, 4/98 e 5/99). Com 4 plantas/parcela a significância do erro 
Tabela 2 - Quadrados médios para blocos, cultivares, erros experimental e amostral da altura de plantas de algodoeiro, obtidos das análises de variâncias individuais de cinco experimentos conduzidos em duas safras agrícolas. EPAMIG/CTNM. Nova Porteirinha, MG, 2000.

\begin{tabular}{|c|c|c|c|c|c|c|c|}
\hline \multirow{2}{*}{$\begin{array}{l}\text { Exp./ } \\
\text { Safra }\end{array}$} & \multirow[t]{2}{*}{ F.V. } & \multirow[t]{2}{*}{ G.L. } & \multicolumn{5}{|c|}{$\mathrm{N}^{\mathrm{o}}$ de plantas amostradas na parcela (n) } \\
\hline & & & 2 & 4 & 6 & 8 & 10 \\
\hline \multirow[t]{4}{*}{$1 / 98$} & Blocos & 3 & $594,11 *$ & $571,02 *$ & $944,71 *$ & $1247,97 *$ & $1357,02 *$ \\
\hline & Cultivares & 3 & $45,03 \mathrm{~ns}$ & $253,64 *$ & $455,49 *$ & $676,38 *$ & $969,02 *$ \\
\hline & Erro experim. & 9 & $105,48 \mathrm{~ns}$ & $227,42 *$ & $327,33^{*}$ & $426,13 *$ & $466,06^{*}$ \\
\hline & Erro amostral & $16(n-1)$ & 46,97 & 79,44 & 89,13 & 83,05 & 78,31 \\
\hline \multirow[t]{4}{*}{$2 / 98$} & Blocos & 3 & $502,21 \mathrm{~ns}$ & $570,06^{*}$ & $792,09 *$ & $949,97 *$ & $1455,62 *$ \\
\hline & Cultivares & 3 & $151,46 \mathrm{~ns}$ & $393,43 *$ & $357,26^{*}$ & $534,20 *$ & $429,85^{*}$ \\
\hline & Erro experim. & 9 & $177,76 \mathrm{~ns}$ & $318,79 *$ & $422,57 *$ & $457,10 *$ & $586,42 *$ \\
\hline & Erro amostral & $16(n-1)$ & 175,75 & 130,67 & 104,71 & 99,53 & 105,12 \\
\hline \multirow[t]{4}{*}{$3 / 98$} & Blocos & 3 & $1098,11^{*}$ & $1529,51 *$ & $1643,18^{*}$ & $1794,00 *$ & $1716,93^{*}$ \\
\hline & Cultivares & 3 & $171,03 \mathrm{~ns}$ & $184,18 \mathrm{~ns}$ & $208,15 \mathrm{~ns}$ & $247,19 *$ & $124,55 \mathrm{~ns}$ \\
\hline & Erro experim. & 9 & $267,30^{*}$ & $431,79 *$ & $724,10^{*}$ & $1044,27 *$ & $1256,88^{*}$ \\
\hline & Erro amostral & $16(n-1)$ & 63,09 & 106,45 & 95,82 & 90,46 & 94,37 \\
\hline \multirow[t]{4}{*}{$4 / 98$} & Blocos & 3 & $56,58 \mathrm{~ns}$ & $34,01 \mathrm{~ns}$ & $204,17 \mathrm{~ns}$ & $504,42 *$ & $614,25 *$ \\
\hline & Cultivares & 3 & $352,25^{*}$ & $477,05 *$ & $644,48^{*}$ & $601,17 *$ & $800,03 *$ \\
\hline & Erro experim. & 9 & $307,55^{*}$ & $598,65^{*}$ & $756,42 *$ & $1017,87 *$ & $1055,47^{*}$ \\
\hline & Erro amostral & $16(n-1)$ & 57,18 & 80,35 & 80,95 & 78,66 & 79,35 \\
\hline \multirow[t]{4}{*}{$5 / 98$} & Blocos & 3 & $106,36 \mathrm{~ns}$ & $199,16^{*}$ & $318,59 *$ & $375,61 *$ & $436,07^{*}$ \\
\hline & Cultivares & 3 & $18,86 \mathrm{~ns}$ & $70,54 \mathrm{~ns}$ & $119,15^{*}$ & $220,38 *$ & $222,14 *$ \\
\hline & Erro experim. & 9 & $75,78 \mathrm{~ns}$ & $172,23 *$ & $249,37 *$ & $273,35 *$ & $233,81 *$ \\
\hline & Erro amostral & $16(n-1)$ & 39,28 & 34,21 & 34,65 & 32,53 & 33,05 \\
\hline \multirow[t]{4}{*}{$1 / 99$} & Blocos & 3 & $235,78 \mathrm{~ns}$ & $259,43 \mathrm{~ns}$ & $500,95^{*}$ & $711,54 *$ & $1141,05^{*}$ \\
\hline & Cultivares & 3 & $265,44 \mathrm{~ns}$ & $453,93 *$ & $765,95^{*}$ & $920,77 *$ & $1468,19 *$ \\
\hline & Erro experim. & 9 & $508,00 \mathrm{~ns}$ & $481,90 *$ & $278,17 \mathrm{~ns}$ & $256,88 \mathrm{~ns}$ & $201,96 \mathrm{~ns}$ \\
\hline & Erro amostral & $16(n-1)$ & 200,53 & 168,68 & 188,26 & 156,03 & 155,95 \\
\hline \multirow[t]{4}{*}{$2 / 99$} & Blocos & 3 & $1190,75^{*}$ & $2751,55^{*}$ & $3277,94 *$ & $3942,52 *$ & $5404,35^{*}$ \\
\hline & Cultivares & 3 & $633,00 *$ & $646,26 *$ & $844,86^{*}$ & $1209,34 *$ & $1556,90^{*}$ \\
\hline & Erro experim. & 9 & $301,80 \mathrm{~ns}$ & $437,16^{*}$ & $455,97 *$ & $416,27 *$ & $452,06 *$ \\
\hline & Erro amostral & $16(n-1)$ & 162,56 & 125,71 & 137,74 & 123,99 & 113,19 \\
\hline \multirow[t]{4}{*}{$3 / 99$} & Blocos & 3 & $703,44 \mathrm{~ns}$ & $1560,55^{*}$ & $2333,59 *$ & $3374,44 *$ & $4254,52 *$ \\
\hline & Cultivares & 3 & $471,53 \mathrm{~ns}$ & $544,26 \mathrm{~ns}$ & $705,56^{*}$ & $901,90 *$ & $1444,73^{*}$ \\
\hline & Erro experim. & 9 & $317,28 \mathrm{~ns}$ & $358,25 \mathrm{~ns}$ & $209,68 \mathrm{~ns}$ & $171,97 \mathrm{~ns}$ & $307,17 \mathrm{~ns}$ \\
\hline & Erro amostral & $16(n-1)$ & 386,09 & 266,56 & 239,18 & 229,46 & 214,19 \\
\hline \multirow[t]{4}{*}{$4 / 99$} & Blocos & 3 & $6849,71^{*}$ & $10917,96 *$ & $12654,84^{*}$ & $15553,68^{*}$ & $19972,04 *$ \\
\hline & Cultivares & 3 & $186,70 \mathrm{~ns}$ & $60,12 \mathrm{~ns}$ & $203,70 \mathrm{~ns}$ & $456,63^{*}$ & $470,92 *$ \\
\hline & Erro experim. & 9 & $366,62 \mathrm{~ns}$ & $549,02 *$ & $618,50^{*}$ & $811,94 *$ & $1060,12 *$ \\
\hline & Erro amostral & $16(n-1)$ & 202,75 & 188,23 & 180,99 & 165,22 & 156,38 \\
\hline \multirow[t]{4}{*}{$5 / 99$} & Blocos & 3 & $1477,37 *$ & $1653,52 *$ & $1836,08^{*}$ & $1287,06^{*}$ & $1291,15^{*}$ \\
\hline & Cultivares & 3 & $1029,45^{*}$ & $1425,27 *$ & $1593,13 *$ & $2046,56 *$ & $1729,07 *$ \\
\hline & Erro experim. & 9 & $567,59 *$ & $903,17 *$ & $786,07 *$ & $823,22 *$ & $817,59 *$ \\
\hline & Erro amostral & $16(n-1)$ & 171,37 & 179,17 & 246,56 & 285,53 & 306,49 \\
\hline
\end{tabular}

${ }^{\text {ns/ }}$ não significativo; ${ }^{* /}$ significativo ao nível de $5 \%$.

experimental foi observada em nove dos dez experimentos, e com 6, 8 e 10 plantas/parcela, em oito, dos dez. Tudo indica que esses resultados sejam reflexos dos acentuados efeitos de 'Blocos' nos experimentos conduzidos, o que também pode ser comprovado pela significância dessa fonte de variação isolada em $86 \%$ das análises. Uma abordagem mais segura, no entanto, poderá ser feita, considerando os coeficientes de variação experimental, por eles estarem ponderados pelo número de plantas amostradas em cada parcela.

Os coeficientes de variação experimental dos experimentos 1/98, 2/98, $3 / 98$ e 4/99 mantiveram-se mais ou menos estáveis com o aumento do número de plantas amostradas na parcela (Tabela 3). Por outro lado, os experimentos 4/98, 5/98, $1 / 99,2 / 99,3 / 99$ e $5 / 99$ apresentaram coeficientes de variação experimental levemente decrescentes com o aumento de plantas na parcela, de acordo com o observado por VENEGAS et al. (1980) com a cultura do café. Essa leve redução dos coeficientes de variação experimental, proporcional ao aumento do número de plantas na parcela, pode ser explicada, devido à menor variabilidade entre as parcelas, quando mais plantas foram consideradas.

Os coeficientes de variação amostral dos experimentos 1/98, 3/98 e 4/98 (Tabela 3) foram mais ou menos estáveis com o aumento do número de plantas amostradas na parcela, enquanto o coeficiente de variação amostral do experimento 5/99 foi crescente. Os coeficientes de variação amostral dos experimentos 2/98, 5/98, 1/99, 2/99, 3/99 e 4/99 sofreram leve redução à medida em que foram aumentadas o número de plantas amostradas na parcela. Analisando-se conjuntamente os coeficientes de variação amostral dos dez experimentos, à medida em que um maior número de plantas foram amostradas na parcela, pode-se inferir que a variabilidade para altura de plantas foi alta. 
Tabela 3 - Coeficientes de variação (C.V.) experimental e amostral da altura de plantas de algodoeiro obtidos das análises de variâncias individuais de cinco experimentos conduzidos em duas safras. EPAMIG/CTNM. Nova Porteirinha, MG, 2000

\begin{tabular}{llrrrrr}
\hline \multirow{2}{*}{$\operatorname{Exp}^{\text {to }}$} & \multirow{2}{*}{ C.V. } & \multicolumn{5}{c}{$\mathrm{N}^{\mathrm{o}}$ de plantas amostradas na parcela (n) } \\
\cline { 3 - 7 } & & \multicolumn{1}{c}{2} & \multicolumn{1}{c}{4} & \multicolumn{1}{c}{6} & \multicolumn{1}{c}{8} & \multicolumn{1}{c}{10} \\
\hline \multirow{2}{*}{$1 / 98$} & Exp. & 7,60 & 9,14 & 9,65 & 10,24 & 9,77 \\
& Amostr. & 9,63 & 13,40 & 14,46 & 14,25 & 13,89 \\
$2 / 98$ & Exp. & 1,52 & 11,14 & 12,02 & 11,26 & 11,89 \\
& Amostr. & 20,14 & 18,57 & 16,91 & 16,80 & 17,57 \\
$3 / 98$ & Exp. & 11,76 & 11,20 & 12,84 & 13,79 & 13,70 \\
& Amostr. & 9,24 & 12,81 & 12,28 & 12,01 & 12,35 \\
$4 / 98$ & Exp. & 14,41 & 14,93 & 14,05 & 14,43 & 13,23 \\
& Amostr. & 9,74 & 11,76 & 11,91 & 11,81 & 11,93 \\
$5 / 98$ & Exp. & 6,65 & 9,20 & 9,35 & 8,62 & 8,59 \\
& Amostr. & 9,76 & 9,16 & 9,20 & 8,96 & 9,01 \\
\multirow{4}{*}{$1 / 99$} & Exp. & 9,75 & 6,88 & 3,01 & 2,78 & 1,68 \\
& Amostr. & 11,13 & 10,10 & 10,67 & 9,78 & 9,79 \\
$2 / 99$ & Exp. & 6,32 & 6,88 & 5,82 & 4,85 & 4,67 \\
& Amostr. & 9,66 & 8,75 & 9,39 & 8,94 & 8,55 \\
$3 / 99$ & Exp. & 4,79 & 4,04 & 1,90 & 2,32 & 2,63 \\
& Amostr. & 16,03 & 13,78 & 13,26 & 13,08 & 12,67 \\
$4 / 99$ & Exp. & 8,89 & 9,39 & 8,46 & 8,99 & 9,42 \\
& Amostr. & 13,98 & 13,56 & 13,34 & 12,85 & 12,40 \\
$5 / 99$ & Exp. & 15,96 & 15,56 & 11,19 & 9,85 & 8,79 \\
& Amostr. & 14,84 & 15,49 & 18,55 & 20,30 & 21,53 \\
& & & & & & \\
\hline
\end{tabular}

Constatou-se também, neste estudo, que os experimentos tiveram boa precisão, devido aos baixos valores dos coeficientes de variação experimental e amostral encontrados. Assim, como síntese das análises realizadas, surge como alternativa para determinação da altura de plantas de algodoeiro, a amostragem de 6 plantas na parcela útil, composta de 2 fileiras de $5 \mathrm{~m}$ de comprimento, em experimentos com 4 repetições instalados em blocos casualizados.

\section{CONCLUSÃO}

A caracterização da altura das plantas de cultivares de algodoeiro herbáceo pode ser feita amostrando-se 6 plantas/parcela, em experimentos de blocos casualizados com quatro repetições.

\section{AGRADECIMENTOS}

Os autores manifestam seus agradecimentos à Fundação de Amparo à Pesquisa do Estado de Minas Gerais (FAPEMIG) e ao Ministério da Ciência e Tecnologia / Conselho Nacional de Desenvolvimento Científico e Tecnológico (MCT/CNPq), pela concessão dos recursos financeiros e bolsas, respectivamente.

\section{REFERÊNCIAS BIBLIOGRÁFICAS}

BARREIRO NETO, M., SOUZA, J.G., BRAGA SOBRINHO, R. Estudo da arquitetura, organografia e do "Shedding" na cultivar Acala Del Cerro (Gossypium hirsutum L. r. latifolium Hutch) tolerantes à seca. In: REUNIÃO NACIONAL DO ALGODÃO, 2, 1982, Salvador, BA. Resumos... Campina Grande : Embrapa, 1982. p.38.

BERTOLUCCI, F. Novas alternativas de tamanho de parcela experimental para avaliação de progênies de feijoeiro. Lavras-MG, 1990. 105p. Dissertação (Mestrado em Genética e Melhoramento de Plantas) - Curso de Pósgraduação em Agronomia Genética e Melhoramento de Plantas, Escola Superior de Agricultura de Lavras, 1990.

CHAVES, L.J., MIRANDA FILHO, J.B. Plot size for progeny selection in maize (Zea mays L). Theoretical and Applied Genetics, Ludhiana, v.84, p.963-970, 1992.

COCHRAN, W.G. Sampling techniques. 3. ed. New York : John Wiley \& Sons, 1977. 428p.

COMISSÃO DE FERTILIDADE DO SOLO DO ESTADO DE MINAS GERAIS. Recomendações para o uso de corretivos e fertilizantes em Minas Gerais. Lavras : UFLA, 1989 . 159p.

CORDEIRO, C.M.T., MIRANDA, J.E.C., CAMPOS, J. Tamanho de parcelas e número de repetições em experimentos de batata. Pesquisa Agropecuária Brasileira, Brasília, v.17, n.9, p.1341-1348, 1982.

FALLIERI, J. Epamig precoce 1: Nova cultivar de algodoeiro para o Norte de Minas. Uberaba : EPAMIG-CTTP, 1994. (EPAMIG-CTTP. Folder de lançamento).

FERH, W.R. Principles of cultivar development. New York : MacMillian, 1987. V.1, 736p.

GOMEZ, K.A., GOMEZ, A.A. Statistical procedures for agricultural research. 2. ed. New York : John Wiley, 1984. 680p.

IGUE, T., SOUZA, M.D., NAGAI, V. Tamanho da parcela mais conveniente para experimentação de campo de arroz. Ciência e Cultura, São Paulo, v.24, n.11, p.1150-1153, 1972.

RAMALHO, M.A.P., FERREIRA, D.F., OLIVEIRA, A.C. Experimentação em genética e melhoramento de plantas. Lavras : UFLA, 2000. 326p.

SANTANA, D.G., OLIVEIRA, M.S., SANTOS JÚNIOR, A.L.G. Comparação entre amostragem de tamanho fixo e amostragem sequencial para avaliação da porcentagem de germinação de sementes de milho (Zea mays L.). Ciência e Agrotecnologia, Lavras, v.21, n.1, p.9-15, 1997.

STEEL, R.G., TORRIE, J.H. Principles and procedures of statistics. New York : McGraw-Hill, 1960. 481p.

VARGAS, M.A. Eficiência da seleção visual e determinação do tamanho de parcela e do número de repetições, para avaliação da produtividade de grãos em famílias $S_{1}$ e $S_{\mathbf{3}}$ de milho (Zea mays L.). Lavras-MG, 1996. 95p. Dissertação (Mestrado em Genética e Melhoramento de Plantas) - Curso de Pós-graduação em Agronomia, Genética e Melhoramento de Plantas, Universidade Federal de Lavras, 1996.

VENEGAS, V.H.A., GUIMARÃES, P.T.G., CARRARO, I.M Efeito da variabilidade de plantas de café dentro da parcela. Revista Ceres, Viçosa, v.27, n.149, p.1-16, 1980. 